\title{
Åland Islands eye disease
}

INSERM

\section{Source}

INSERM. (1999). Orphanet: an online rare disease and orphan drug data base. Aland Islands eye disease. ORPHA:178333

Aland Island Eye Disease (AIED) is an X-linked recessive retinal disease characterized by fundus hypopigmentation, decrased visual acuity, nystagmus, astigmatism, progressive axial myopia, defective dark adaptation and protanopia. 